\title{
Organelle Isolation by Magnetic Immunoabsorption
}

BioTechniques 26:336-343 (February 1999)

\author{
Albert P. Kausch, T. Page \\ Owen, Jr. ${ }^{1}$, S. Narayanswami ${ }^{2}$ \\ and Barry D. Bruce ${ }^{3}$ \\ University of Connecticut, \\ Storrs, ${ }^{1}$ Connecticut College, \\ New London, CT, ${ }^{2}$ The Jackson \\ Laboratory, Bar Harbor, ME, \\ ${ }^{3}$ University of Tennessee, \\ Knoxville, TN, USA
}

\section{INTRODUCTION}

Most procedures for subcellular fractionation exploit size and density differences between the various organelles and intracellular structures, and utilize centrifugation for separation (24). Unfortunately, many cellular components are difficult or impossible to isolate to purity by density sedimentation or other traditional fractionation methods. The viability of using magnetic immunoabsorption to immobilize organelles has been demonstrated (9), and higher plant chloroplasts have been successfully recovered from whole cell lysates (11). Chloroplast isolation was accomplished with biotinylated antibodies raised against the chloroplast outer envelope and magnetic nano-particles coupled to streptavidin. Magnetically labeled organelles were separated on a ferromagnetic affinity column placed in a high-gradient magnetic field and recovered when the column was removed from the magnetic field and eluted with an isotonic buffer. However, this procedure's applicability is limited because it is not easily modified for small sample volumes and can only be applied to one sample at a time.

Magnetic immunoabsorption of organelles requires that targeted epitopes are readily accessible to biotinylated antibodies, as Figure 1 schematically demonstrates. The number of exposed epitopes and the strength of the antigen/antibody interaction determine the efficacy of this technique. Obviously, not all antibodies to organellar proteins will be effective in this approach. However, antibodies specific to external epitopes on several intracellular compartments and structures have been reported $(5,19,21,28)$, and their specificity has been demonstrated immuno- cytochemically.

Since the first magnetic cell purifications were achieved several years ago, various different magnetic particle preparations have been developed (8). These preparations have distinct magnetic properties, surface characteristics and mean diameter ranges. Their physical and chemical differences significantly influence their utility for subcellular isolations. These preparations usually can be divided into two major classes based on size: microparticle preparations ( $\mu \mathrm{m}$ in diameter) and nanoparticle preparations ( $\mathrm{nm}$ in diameter).

Here, we utilize both magnetic microparticles and nanoparticles to immobilize and recover various subcellular fractions. First, we demonstrate that both types of particle successfully label subcellular organelles. Although not ideal for purification, microparticles are very effective in the immobilization and manipulation of purified cellular components. We show that magnetic nanoparticles are useful for isolation of

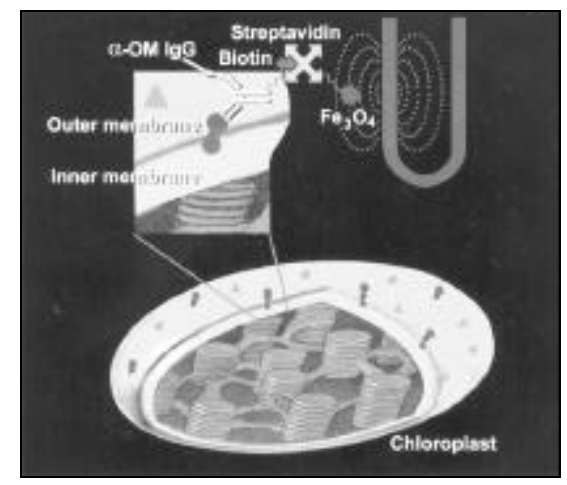

Figure 1. A schematic representation of indirect immunolabeling of chloroplasts with biotinylated antibody preparations and streptavidin magnetic particles. The U-shaped structure represents the steel-collecting loop with induced magnetic field from the internal-field magnetic collecting device. 
subcellular components, including plastids and nuclei, from whole cell lysate. Organelles labeled with magnetic nanoparticles must be separated either in a magnetic affinity column as demonstrated previously $(11,16)$ or an internal-field, high-gradient magnetic separation device (15). An internal magnetic separation device allows for simultaneous processing of several samples for direct comparison and quantitation in microarrays. The combined use of nanoparticles and internal high field separation allows simultaneous processing of ten different samples. This procedure provides not only intact, purified organelles, but has also proven to be quantitative in recovery. The use of the microarray format enables isolations to be done under conditions where the starting material is limiting or otherwise restricted to $100 \mu \mathrm{L}$ or less. In addition, it permits a rapid and versatile analysis using a variety of different microplate readers (including absorbance, fluorescence and scintillation detection) and therefore enables high-throughput experimental design.

\section{MATERIALS AND METHODS}

\section{Immunolabeling Reactions}

To prepare whole plant cell lysates, plant cell protoplasts were isolated according to the procedure by Walbot (27) from pea leaf mesophyll, excised maize endosperm and Black Mexican Sweet (BMS) maize suspension culture cells, and broken through a $37-\mu \mathrm{m}$ mesh nylon screen directly into the appropriate immunolabeling buffer and incubated 5-20 $\mathrm{min}$ at $4^{\circ} \mathrm{C}$. The mouse metaphase chromosomes were prepared as previously described (18). Nuclei suspensions were prepared according to the method of Stoppin et al. (25) from maize BMS protoplasts. Separate immunolabeling buffers were prepared from isolation buffers for chloroplasts (11), amyloplasts (11), chromosomes $(3,12,18)$ and nuclei $(25)$ with the addition of $2 \%$ nonfat milk, $0.5 \%$ bovine serum albumin (BSA), $20 \mathrm{mM} \mathrm{KCl}$ and $0.05 \mathrm{mg} / \mathrm{mL}$ nonimmune serum. These preparations were either labeled directly by the addition of coupled antibody/magnetic particle solution or pre-

Table 1. Antibodies Used for Organelle Isolation

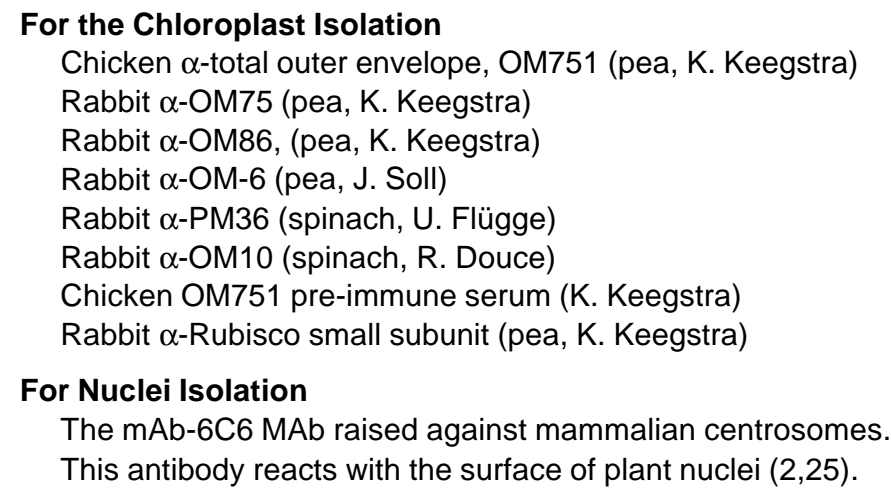

pared for secondary labeling by the addition of the biotinylated antibody. The antibody concentration varied between $10-250 \mu \mathrm{g} / \mathrm{mL}$.

\section{Antibody and Protein Preparations}

The antibodies used were in the form of unfractionated serum (see Table 1). Antibody preparations were biotinylated with EZ-Link ${ }^{\mathrm{TM}}$ NHS-LCBiotin (Pierce Chemical, Rockford, IL, USA) and tested with streptavidin-coated particles.

\section{Preparation of Magnetic Microparticles}

Magnetic microparticles were prepared from affinity-chromatographygrade BioMag (Advanced Magnetics, Cambridge, MA, USA). BioMag magnetic microparticles were first densitysedimented to recover particles that were $<0.5-\mu \mathrm{m}$ average diameter, then rinsed with acetone and refluxed for 24 $\mathrm{h}$ with $N$-(2-aminoethyl)-3-aminopropyltriethoxysilane, providing an amino functional surface to which the antibody preparations were covalently coupled directly using reversible and nonreversible, Homobifunctional CrossLinking Reagents (Pierce Chemical). Superparamagnetic polymer microspheres, $0.74 \mu \mathrm{m}$, (Bangs Laboratories, Fishers, IN, USA) were covalently coupled to antibody and streptavidin by amino linkages. Coupling efficiency for streptavidin was determined using an HRP-biotin conjugate titration series (maximum titer of conjugate per unit mass of particle) in an assay using ortho-phenylenediamine (OPD) color-de- veloping substrate read after binding and washing of unbound conjugate at $492 \mathrm{~nm}$ after termination with sulfuric acid. Assays were performed in a similar manner for other protein-particle conjugates. Antibody preparations were biotinylated with NHS-LC-biotin (Pierce Chemical) and tested with streptavidin-coated particles. Control samples were incubated with various nonimmune magnetic antibody preparations, which were constructed in a similar fashion. Streptavidin-conjugated particles were obtained from the manufacturers listed in Table 2 . Amounts of streptavidin and magnetic compositions were provided by the manufacturer.

\section{Magnetic Separation Devices}

Magnetic affinity columns can be purchased commercially (Becton Dickinson Labware, Bedford, MA, USA) or constructed using ferromagnetic steel wool as the matrix (11). Mini-MACS columns were from Miltenyi Biotech (Auburn, CA, USA). The high-gradient, internal-field magnetic separator and protein separator were designed by Immunicon (Huntingdon Valley, PA, USA). Neodymimium-iron-boron disc magnets and cell separators were from Advanced Magnetics and Dynal (Lake Success, NY, USA), respectively.

\section{Testing of Various Antibodies for Isolation of Chloroplasts in Microwells}

Immunolabeling reactions on pea chloroplasts contained $50 \mu \mathrm{g}$ chlorophyll, $50 \mu \mathrm{L}$ blocking buffer (2\% non- 
fat milk, $20 \mathrm{mM} \mathrm{KCl}$ and $0.05 \mathrm{mg} / \mathrm{mL}$ pre-immune serum [PIS] in chloroplast isolation buffer) with various amounts of biotinylated antisera (each antisera was adjusted to $2 \mathrm{mg} / \mathrm{mL}$ total protein) diluted with isolation buffer to $300 \mu \mathrm{L}$ final volume and were conducted for 20 min on ice in strips of microwells. The labeled chloroplasts were then reacted for 10 min with $20 \mu \mathrm{L}$ of direct-coat streptavidin magnetic nanoparticles. The optimum rates for chloroplast recovery were determined by Kausch and Bruce (11). Separation is achieved by inserting the ferromagnetic pins into the microwells in a high-gradient magnetic field (0.6 Tesla).

\section{Light Microscopy}

Light micrographs of isolated organelles were taken on samples in isolation buffer immediately after isolation using phase contrast, differential interference contrast or fluorescence microscopy. Chromosomes were stained with propidium iodide immediately before photomicroscopy. Isolated nuclei samples were fixed in Karnovsky's fixative for $1 \mathrm{~h}$, post-fixed in $2 \%$ osmium tetroxide in $50 \mathrm{mM}$ potassium phosphate buffer ( $\mathrm{pH}$ 7.2) for $1 \mathrm{~h}$, dehydrated in an ethanol series and embedded in Spurr's resin. Sections (1.0-2.0 $\mu \mathrm{m})$ were stained with methylene blue and photographed with phase-contrast optics.

\section{Flow Cytometry of Nuclei}

After nuclei were purified by magnetic immunoabsorption, they were stained with propidium iodide. After staining, 10000 nuclei were analyzed in a FACStar ${ }^{\text {PLus }}$ Flow Cytometer (Becton Dickinson, Immunocytometry Systems, San Jose, CA, USA). Cell cycle analysis was done using the ModFitLT ${ }^{\mathrm{TM}}$ V1.00 program (Verity Software House, Topsham, ME, USA).

\section{RESULTS AND DISCUSSION}

Evaluation of Directly Labeled Magnetic Microparticles' Ability to Specifically Recover Organelles

Positive selection of rare cells has been successfully accomplished using

Table 2. Commercially Available Streptavidin-Coupled Magnetic Particle Preparations

\begin{tabular}{|ll|}
\hline Manufacturer & Type of Preparation \\
\hline $\begin{array}{l}\text { 1. Bioquest, Raleigh, NC, USA } \\
\text { 2. Bangs Laboratory }\end{array}$ & $\begin{array}{c}\text { Nanoparticle } \\
\text { Nanoparticle and } \\
\text { microparticle }\end{array}$ \\
3. Immunicon & Nanoparticle \\
4. Miltenyi Biotech & Nanoparticle \\
5. Advanced Magnetics & Microparticle \\
6. PerSeptive Biosystems, Framingham, MA, USA & Microparticle \\
7. Dynal & Microparticle \\
\hline
\end{tabular}

direct immunolabeling with magnetic microparticles $(14,17,20,23)$. To evaluate this approach for organelle isolation, we directly labeled two types of magnetic microparticles with organelle-specific antibodies. These two particle types, superparamagnetic polymer microparticles (Bangs Laboratories) and silane-treated affinity chromatography BioMag particles (Advanced Magnetics), were compared in regards to their ability to immunoabsorb either higher plant plastids or (mouse) chromosomes. In addition, to confirm the specificity of this approach, we utilized several antibody preparations, including those that should provide positive selection for plastids $(\alpha$-OM751, $\alpha$ OEP86 and $\alpha$-OEP75), positive selection for chromosomes (anti-histone $\alpha$ HIS), $\alpha$-OM751 (PIS) and several control antibodies that should fail to react with either chloroplasts or chromosomes (Figure 2).

Precautions were taken to assure that, in all cases, the magnetic particles were coated to the same extent with functional proteins. This was confirmed by colorimetric detection using the appropriate HRP-conjugated secondary antibodies and OPD/peroxide substrates (data not shown). In all cases, the binding capacity on both types of microparticles were very similar for the different antibody preparations and was calculated to be between $0.15-1.0 \mu \mathrm{g}$ $\mathrm{IgG} / 10^{6}$ particles. Consistent with previous results on organelle isolation with magnetic microparticles (9), when microparticles were tested for their rate of reactivity towards plastids and (mouse) metaphase chromosomes, both sizes were quite inefficient in their ability to recover organelles when the incubation time was less than $10 \mathrm{~min}$. Although low numbers of organelles can be recovered as is shown in Figure 2, a-c, recovery is less than $1 \%-2 \%$, and most of the microparticles are recovered alone as small aggregates. The use of antisera for direct immunomagnetic purification of organelles is expected to result in low rates of recovery due to low relative contribution of specific IgG protein to total serum protein. Upon extended incubation (>60 min), large numbers of pre-isolated pea chloroplasts do become magnetically responsive and can be immobilized on a neodymimiumiron boron disc magnet (Figure 2d). Even though this interaction is largely nonspecific, since both $\alpha$-HIS or $\alpha$ OM751 PIS react strongly with chloroplasts after an equally long incubation (data not shown), these microparticle preparations can provide a rapid and gentle means of organelle recovery and manipulation that can be done without centrifugation or other disruptive treatments. Therefore, this approach can be valuable in experiments that need to be able to rapidly change the composition of the resuspension buffer, as for example, studies on protein import or metabolic pathways.

\section{Recovery and Quantitation of Organelles in Microarrays}

We screened eight different commercially available streptavidin-coupled magnetic particle preparations (see Table 2) including four different microparticle preparations and four different nanoparticle preparations. In all cases, the organelle recovery involved indirect positive selection by means of a streptavidin/biotin interaction and a fixed amount of biotinylated $\alpha$-OM751 antibody (11). We found that magnetic 
nanoparticles provided the most specific and efficient recovery of chloroplasts and developed a new system for organelle immunoabsorption based on streptavidin direct-coat magnetic nanoparticles attached to specific organelles by a biotinylated antibody and isolation by internal field separation. Recovery is accomplished in microarrays, which are placed in the internalfield magnetic separation device shown in Figure 3. This device utilizes a row of ferromagnetic loops that are inserted into microwells within a high-gradient magnetic field (0.6 Tesla). The labeled organelles magnetically adhere to the loops. Absorbed organelles are removed with the loop from the magnetic field and released when placed in a second microarray containing fresh isolation buffer, which promotes their release. The recovery of chloroplasts can then be accurately and conveniently measured in a microplate reader at 650 $\mathrm{nm}$. This system provides a rapid, easy method of comparing the efficacy of various antibodies and the effect of an- tibody dilution on organelle recovery. Immobilization of organelles also provides the possibility of quantification of in vitro organelle reactions, such as protein import and in organellar protein synthesis, where these reactions can be conducted in microwells, and wash steps are accomplished without deleterious centrifugation.

\section{Identification of Effective Outer Membrane Epitopes for Magnetic Immunoabsorption of Chloroplasts}

To compare the effect of antibody dilution and the utility of various antibodies to pea and spinach proteins for chloroplast recovery, a dilution series of immunoreactions were made in microwells using one of the following biotinylated antibodies: $\alpha$-OM751 (pea), PIS (OM751), $\alpha$-OEP75 (pea), $\alpha$-OEP86 (pea), $\alpha$-OEP (pea), $\alpha$-E10 (spinach), $\alpha-\mathrm{P} 36$ (spinach) or $\alpha-\mathrm{SSU}$ (pea). Since $\alpha-S S U$ recognizes a stromal protein, it should function like the PIS, as a negative control for recovery

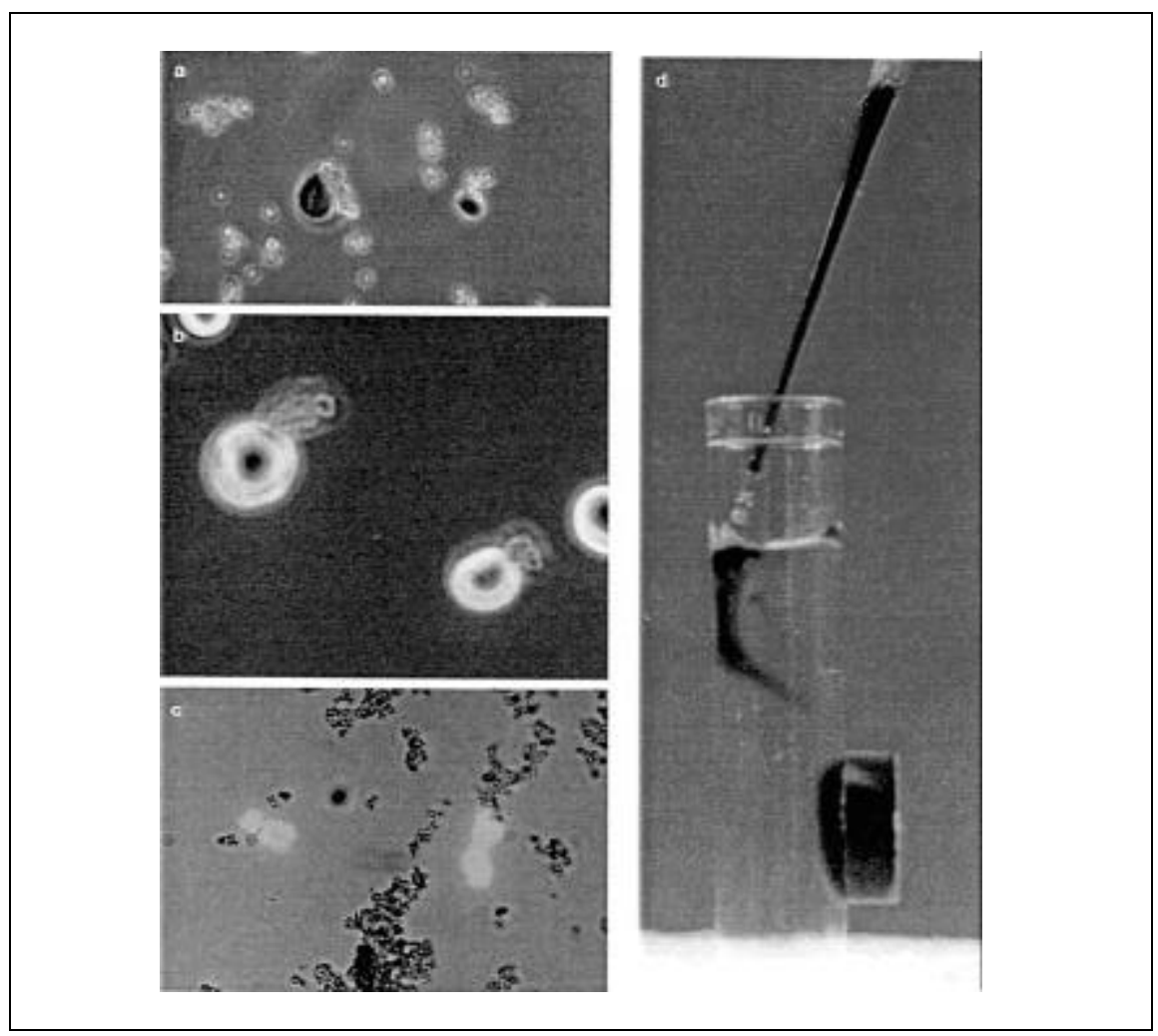

Figure 2. Magnetic immunoabsorption of organelles to microparticles by direct labeling. BioMag particles immunolabeled the following: (a) pea chloroplasts, (b) maize amyloplasts and (c) mouse (L929) metaphase chromosomes after 10-min incubation. (d) Immobilization of pea chloroplasts on a neodymimium-iron boron disc magnet after extended incubation. 
of intact plastids (7). The antibody $\alpha$ P36 was raised against an inner envelope membrane phosphate translocator and therefore should also not react with intact chloroplasts (4). The antibodies $\alpha$-OM751 (11), $\alpha$-OEP75 $(22,26), \alpha$ OM86 $(22,24)$ and $\alpha$-E10 $(1,10)$ have all been shown to localize to proteins in the outer chloroplast membrane. Three of these antibodies, $\alpha$-OM751, $\alpha$ OEP75 and $\alpha$-OEP86, were raised against pea chloroplast proteins, whereas $\alpha$-E10 is a monoclonal antibody (MAb) to a spinach chloroplast protein.

Figure $4 \mathrm{~A}$ shows a photograph of a typical recovery profile, which demonstrates the interaction of all of the antibodies tested against spinach chloroplasts. The amount of chlorophyll in these wells was quantitated in the plate reader at $650 \mathrm{~nm}$ and is plotted in Figure 4B. The rates of chloroplast recovery in these experiments were those found to be optimal in previous work by Kausch and Bruce (11). As expected, antibodies that react with internal and therefore inaccessible epitopes (SSU or P36), fail to recover chloroplasts even at high concentrations. Surprisingly, two of the antibodies tested react with outer

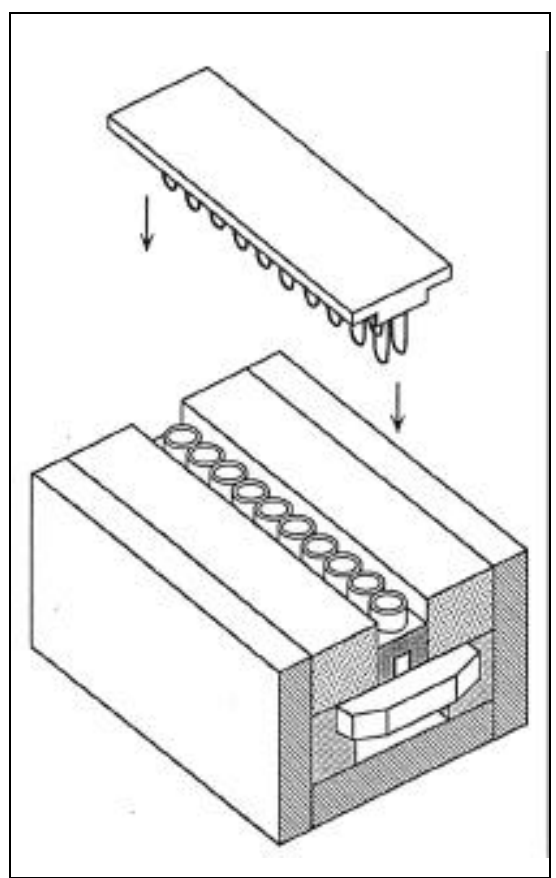

Figure 3. Schematic of internal-field, high-gradient magnetic separator. Shown with microstrips in place within the magnetic field. The steel-collecting loops are shown above and can be easily inserted into each well of the microstrip. envelope epitopes, $\alpha$-OEP75 and $\alpha$ OM86, fail to provide effective recovery of either pea or spinach chloroplasts. However, $\alpha$-OEP75 does appear to pro-

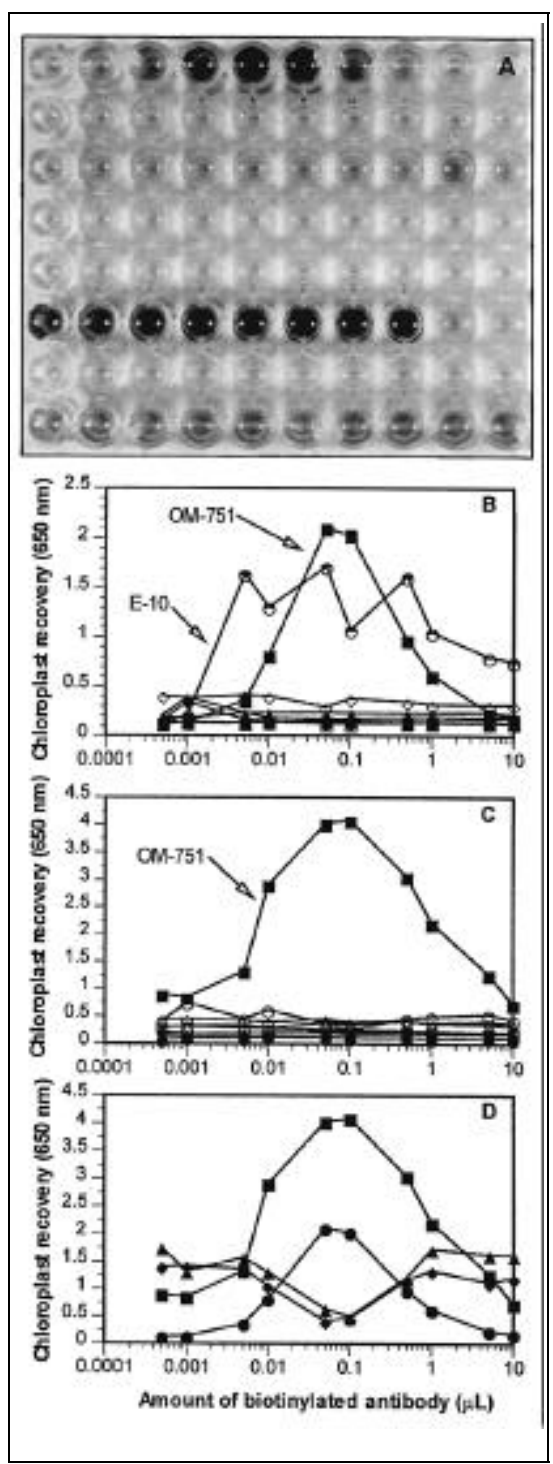

Figure 4. Internal field quantitative organelle recovery in microarrays. Comparison of dilution series of various antibodies to chloroplast proteins for magnetic immunoabsorption. (A) Photograph of typical microwell plate containing magnetically isolated spinach chloroplasts after magnetic separation in response to an antibody dilution series. Antisera tested are from the top to bottom: anti-total outer envelope, $\alpha$-OM751; $\alpha$ OM75; $\alpha$-OM86; $\alpha$-OM-6; $\alpha$-IM36; $\alpha$-OMI0; OM751 PIS; and $\alpha$-Rubisco small subunit. (B) Quantitiation of the of microplate titration are shown above using intact pea chloroplasts and (C) using intact spinach chloroplasts. (D) A comparison of magnetic immunoabsorption for pea (squares) and spinach (circles) chloroplasts and their corresponding depletion (diamonds and triangles) using biotinylated $\alpha$-OM751. vide a low level of recovery in our assay. Only $\alpha$-OM751 and $\alpha$-E10 are capable of magnetically immunoabsorbing chloroplasts. When the same analysis was performed with pea chloroplasts, only $\alpha$-OM751 was found to be effective for magnetic recovery of

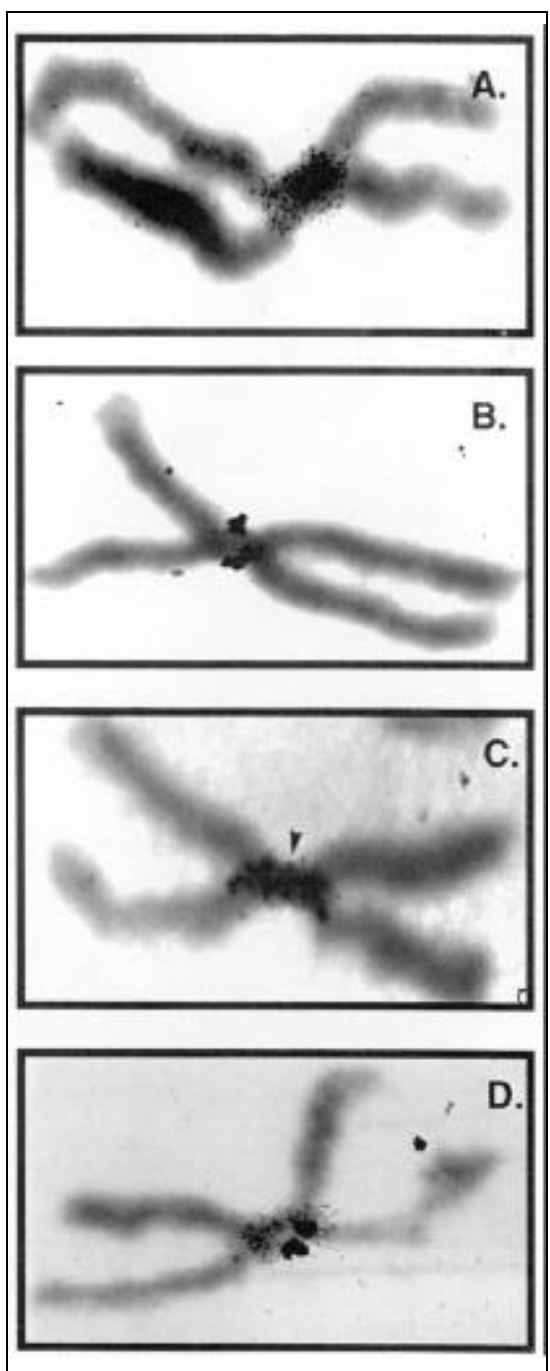

Figure 5. Labeling of mouse metaphase chromosomal centromeres with magnetic particles. (A) Mouse L939 cell metaphase chromosome after in situ hybridization with biotin-labeled pSAT1 DNA followed by detection with streptavidin-labeled, 20-nm colloidal gold. (B) L929 cell chromosome as in Panel A after hybridization with pSAT1 and direct labeling with BioMag microparticles. Specific labeling of the centromere is observed. (C) Chromosome as in Panel A after labeling with streptavidin-conjugated nanoparticles. High levels of labeling of the centromere and negligible nonspecific labeling are observed. (D) Chromosome labeled with colloidal gold followed by magnetic microparticles. Magnetic particles are seen only in regions previously labeled with gold. 
chloroplasts from both plants (Figure $4 C)$. Interestingly, since $\alpha$-E 10 is only able to recover chloroplasts from spinach (Figure 4, B and C), the epitopes that are recognized by $\alpha$-E10 appear not to be conserved between spinach and pea. In all cases, the integrity of the recovered chloroplasts was quite high as determined by visual inspection by Nomarski interference contrast microscopy and density sedimentation in Percoll ${ }^{\circledR}$ gradients (data not shown).

The unique ability of $\alpha$-OM751 to provide a quantitative means of recovering chloroplasts is probably the result of this antibody preparation having been raised against the entire outer envelope (13). This antigen not only provides multiple different epitopes, but it also retains epitopes in a native or near-native state. Although the minimum required number of immunoabsorbed magnetic nanoparticles per organelle for recovery has not been determined for this type of collection device, it is potentially greater than the number of available target epitopes for $\alpha$-OM75 and $\alpha$-OM86. These two proteins have been shown recently to be components of the chloroplast protein translocation apparatus $(22,24,26)$. It has also been shown that this complex is only present in approximately 2300 copies per chloroplast (6). Alternatively, the majority of the epitopes that these antibodies recognize might not be fully exposed in intact chloroplasts and might therefore be nonreactive for the purposes of immunolabeling. This is supported by reports that these antibodies only partially inhibit protein transport, even when tested at extremely high levels $(24,26)$. The recovery of chloroplasts with $\alpha$-SSU was higher than expected, because this antibody recognizes an internal stromal protein. However, the holoenzyme Rubisco is very abundant and might be cross-contaminating intact plastids by nonspecifically absorbing to their exteriors after being released from other plastids during cell disruption.

\section{Magnetic Labeling of Chromosomes by In Situ Hybridization}

We used magnetic labeling of chromosomes after in situ hybridization with a biotinylated DNA probe to explore the specificity of labeling that can be obtained with various streptavidin-coated magnetic particles. Colchicine-treated mouse L929 cells were used to prepare Miller-type chromosome spreads on carbon-evaporated, parlodion-coated gold electron microscopy (EM) grids. Mouse major centromeric satellite DNA (pSAT 1) (18) was biotinylated by nick-translation and hybridized to the chromosomes by standard methods (18). As a control, streptavidin-conjugated colloidal gold $(20 \mathrm{~nm})$ was used to label the in situ hybridized chromosomes, and preparations were visualized by EM (Figure 5A). We were able to demonstrate specific labeling of L929 cell chromosomal centromeres with both streptavidin BioMag microparticles (Figure 5B) and streptavidin-conjugated nanoparticles (Figure 5C). Levels of nonspecific labeling were consistently low. In controls labeled with both gold and magnetic particles, the latter were found only in regions previously labeled with gold (Figure 5D). Our results demonstrate that magnetic particles can be used to label chromosomes with high specificity and signal:noise ratios. We found that once the chromosomes are deposited on a solid support such as a coated EM grid or glass slide and processed through the in situ hybridization reactions, they could not be removed by conventional procedures. However, it might be possible to digest magnetically labeled chromosomes with restriction endonucleases for recovery of specific labeled sequences or chromosome fragments.

\section{Isolation and Immobilization of Nuclei by Magnetic Immuno- absorption}

The need to purify nuclei from higher plants is necessary for studies in the regulation of gene expression, RNA processing and transport, and nuclear protein trafficking. Typical nuclei preparations from maize BMS cells by density sedimentation (at $1 \mathrm{~g}$ ) are contaminated with cellular components such as amyloplasts and starch grains (25). We tested whether the MAb mA-6C6 would be effective for immune-isolation and immobilization of maize BMS nuclei from density-sedimented preparations and whole cell 
lysates. This MAb was raised against a mammalian pericentriolar antigen and recognizes plant microtubule-organizing centers on the surface of the nuclear envelope $(2,25)$, and it was chosen because it has been shown to immunolocalize to the outside surface of maize
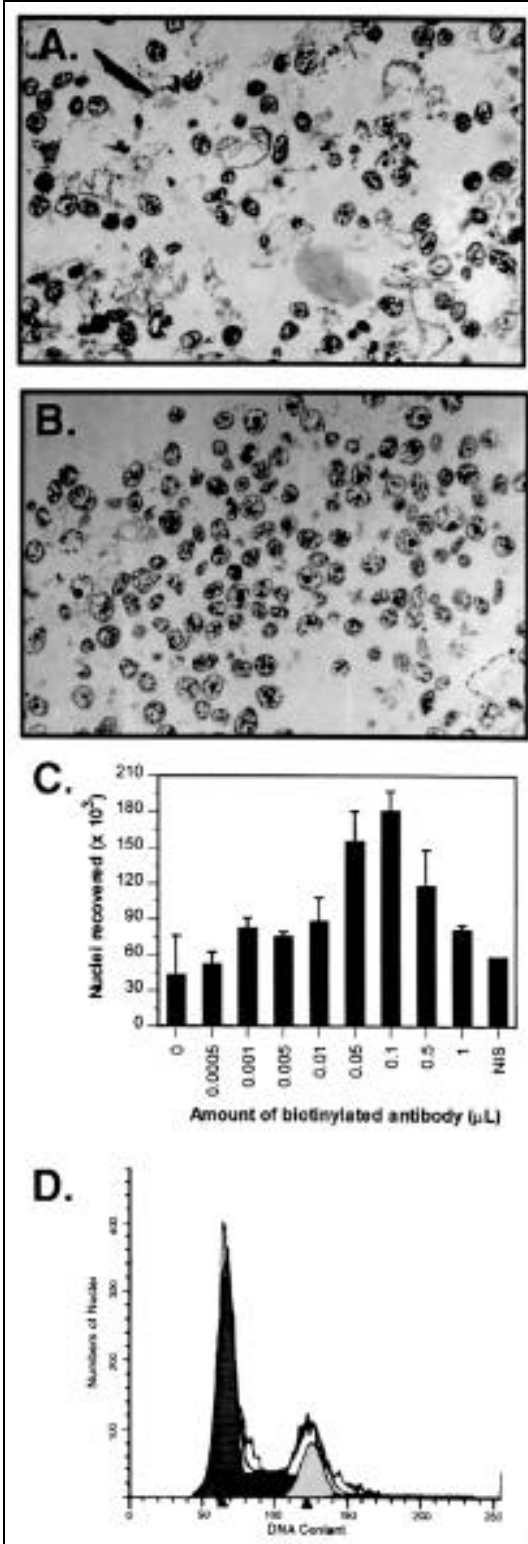

Figure 6. Isolation of nuclei by magnetic immunoabsorption. (A) Phase contrast of $1.5-\mu \mathrm{m}$, methylene blue-stained thick sections through pellets of density-isolated BMS nuclei and (B) magnetically separated nuclei. (C) Titration of recovery of nuclei from a dilution series with biotinylated mAb-6C6. (D) FACS analysis of magnetically isolated nuclei. These nuclei were stained with propidium iodide. Cell cycle analysis was done using the ModFitLT V1.00 program.
BMS suspension culture nuclei (25).

Initially, we screened various particle types, magnetic separation devices and immunolabeling reactions for nuclei isolation. Because of clumping and aggregation difficulties associated with most approaches, we chose to label nuclei with biotinylated mAb-6C6 and streptavidin-conjugated magnetic nanoparticles (Miltenyi Biotech) and to recover them through a magnetic affinity column. To determine the optimum antibody concentration, the nuclei samples were incubated with a dilution series containing $0-500 \mu \mathrm{g}$ of the biotinylated mAb-6C6. After labeling for $10 \mathrm{~min}$ at $4^{\circ} \mathrm{C}$, the nuclei were then reacted for $15 \mathrm{~min}$ with $100 \mu \mathrm{L}$ of conjugated nanoparticles and separated on a column adjusted to a flow rate of 200 $\mu \mathrm{L} / \mathrm{min}$ at $4^{\circ} \mathrm{C}$ in a high-gradient magnetic field. After washing the column with approximately $10 \mathrm{vol}$ of isolation buffer at a flow rate of $0.75 \mu \mathrm{L} / \mathrm{min}$, the column was removed from the magnetic field and gently back-flushed twice with $1.0 \mathrm{~mL}$ cold isolation buffer. The back-flushing and reabsorption step free nonspecific material physically trapped in the column matrix. The separated nuclei were eluted from the column matrix after removal from the magnetic field and back-flushing with cold isolation buffer. Figure 6 shows micrographs of the starting material (Panel A) vs. the magnetic immunopurified nuclei (Panel B). The immunopurified nuclei are substantially concentrated and largely free from the cellular debris in the starting material.

The optimum antibody concentration was determined by the number of nuclei recovered and plotted as a function of the biotinylated mAb-6C6 used during isolation (Figure 6C). With the isolation parameters described above, only low numbers of nuclei are retained on the column either in the absence of biotinylated antibody (far left column) or when biotinylated nonimmune serum (NIS) is used (far right column). The number of nuclei recovered increases with an optimum of $250 \mu \mathrm{g} / \mathrm{mL}$ biotinylated mAb-6C6. We routinely recover $1.8 \times 10^{4}$ nuclei $/ \mathrm{mL}$ using this procedure, as is shown in Figure 6B. The purity, integrity and cell-cycle distribution of the magnetically immunoabsorbed nuclei was evaluated by flow cytometry. After staining the nuclei with propidium iodide, $10000 \mathrm{nu}$ clei were analyzed in a flow cytometer. Figure 6D shows the histogram of the DNA fluorescence. Analysis of this histogram indicates that $77.3 \%$ of the nuclei were diploid, $22.7 \%$ were tetraploid and $31.9 \%$ total were in Sphase. In addition, this fluorescenceactivated cell sorting (FACS) analysis indicated that magnetically immunoabsorbed nuclei contained only $3.25 \%$ debris and no aggregates. The cell cycle analysis also reveals that magnetic separation with this antibody does not select for specific cell-cycle populations of BMS nuclei.

\section{Advantages of Organelle Isolation by Magnetic Immunoabsorption}

In this paper, we have demonstrated that biotinylated antibodies specific to exposed epitopes on subcellular structures can be used for immuno-isolation and immobilization of various organelles. We examined several preparations of magnetic particles with distinct magnetic properties, surface characteristics and mean diameter-size ranges for their specific utility for subcellular isolations or immobilizations. Both microand nanoparticles are capable of labeling subcellular organelles in a highly specific manner, and the various particles have distinct applications. Organelle isolation from whole cell lysates is possible with magnetic nanoparticles largely because of the properties of the particles and the strength of the magnetic collection device used for recovery. Internal-field magnetic separation is very efficient for absorption and collection of organelles labeled with magnetic nanoparticles (ca. $120 \mathrm{~nm}$ ). The internal-field magnetic separator also provides a method for quantitative recovery of labeled structures in microarrays, a distinct advantage in processing a number of samples simultaneously. This allows immobilization of organelles and provides the possibility for direct quantitation of in vitro organelle reactions, such as in organellar protein synthesis, protein import and or nuclear run-on experiments, where these reactions can be conducted in microwells, and wash steps are accomplished without deleterious centrifugation. 


\section{ACKNOWLEDGMENTS}

We thank Drs. Jerry Marshall, Ken Keegstra, Jürgen Soll, Roland Douce, Barbara Hamkalo, Ann Lambert and Ingo Flügge for their generous gifts of the antibodies used in this project and Drs. Paul Liberti and Brian Feeley at Immunicon for providing the diagram and use of their internal field magnetic separator and magnetic particles. We also thank Bioquest, Perseptive Biosystems, Bangs Laboratory and Ferrofluid Inc. for their generous gifts of magnetic particles, Dr. A. Cunningham (Pfizer, Inc., Groton, CT, USA) for her assistance in the FACS analysis and Ms. Julie Biggs, Connecticut College, for her technical assistance. This work was supported by a National Science Foundation (NSF) Cell Biology Program Grant (MCB 9401840) to B.D.B.

\section{REFERENCES}

1.Block, M.A., A.J. Dorne, J. Joyard and R. Douce. 1983. Preparation and characterization of membrane fractions enriched in outer and inner envelope membranes from spinach chloroplasts. I. Electrophoretic and immunochemical analysis. J. Biol. Chem. 258:1327313280.

2.Chevrier, V., S. Komesli, A.-C. Schmit, M. Vantard, A.M. Lambert and D. Job. 1992. A monoclonal antibody raised against mammalian centrosomes and screened by recognition of plant microtubule-organizing centers identifies a pericentriolar component of different cell type. J. Cell Sci. 101:823-835.

3.Dudin, G., E.W. Steegmayer, P. Vogt, H. Schnitzer, E. Diaz, K.E. Howell, T. Cremer and C. Cremer. 1988. Sorting of chromosomes by magnetic separation. Human Genet. 80:111-116.

4.Flügge, U.I., K. Fischer, A. Gross, W. Sebald, F. Lottspeich and C. Eckerskorn. 1989. The triose phosphate-3-phosphoglycerate-phosphate translocator from spinach chloroplasts: nucleotide sequence of a full-length cDNA clone and import of the in vitro synthesized precursor protein into chloroplasts. EMBO J. 8:39-46.

5.Franzusoff, A., E. Lauze and K.E. Howell. 1992. Immuno-isolation of Sec7p-coated transport vesicles from the yeast secretory pathway. Nature 331:232-234.

6.Friedman, A. and K. Keegstra. 1989. Chloroplast protein import: quantitative analysis of precursor binding. Plant Physiol. 89:993-999.

7.Hartman, F.C. and M.R. Harpel. 1994. Structure, function, regulation, and assembly of D-ribulose-1,5-bisphosphate carboxylase/ oxygenase. Annu. Rev. Biochem. 63:197-234.

8.Haukanes, B.-I. and C. Kvam. 1993. Application of magnetic beads in bioassays. Bio/Technology 11:60-63.
9.Howell, K.E., R. Schmid, J. Ugelstad and J. Gruenberg. 1989. Immunoisolation using magnetic solid supports: subcellular fractionation for cell-free functional studies. Methods Cell Biol. 31:256-292.

10.Joyard, J., A. Billecocq, S.G. Bartlett, M.A. Block, N.H. Chua and R. Douce. 1983. Localization of polypeptides to the cytosolic side of the outer envelope membrane of spinach chloroplasts. J. Biol. Chem. 258:10000-10006.

11.Kausch, A.P. and B.D. Bruce. 1994. Isolation and immobilization of various plastid subtypes by magnetic immunoabsorption. Plant $\mathrm{J}$. 6:767-779.

12.Kausch, A.P. and S. Narayanswami, inventors; Dekalb Genetic Corporation, assignee; Isolation of biological materials. USA. Patent No. 5,665,582. 1997 September 9.

13.Keegstra, K. and Y. Abdullah. 1986. Isolation and characterization of chloroplast envelope membranes. Methods Enzymol. 118:316325.

14.Leach, L., Y. Bhasin, P. Clark and J.A. Firth. 1994. Isolation of endothelial cells from human term placental villi using immunomagnetic beads. Placenta 15:355-364.

15.Liberti, P.A. and B.P. Feeley. 1991. Analytical and process scale cell separation with bioreceptor ferrofluids and high-gradient magnetic separation, p. 268-288. In D.S. Kompala and P. Todd (Eds.), Cell Separation Science and Technology, Vol 464. American Chemical Society, Washington, DC.

16.Molday, R.S., S.P.S. Yen and A. Rembaum. 1989. Application of magnetic microspheres in labeling and separation of cells. Nature 268:437-438.

17.Mul, F.P.R., E.F. Knol and D. Roos. 1992. An improved method for purification of basophilis granulocytes from human blood. J. Immunol. Methods 149:207-214.

18.Narayanswami, S., K. Lundgren and B.A. Hamkalo. 1989. Deoxyribonucleic acid sequence mapping on metaphase chromosomes by immunoelectron microscopy. Scan. Electron Microsc. 3:65-76.

19.Ohba, M. and G. Schatz. 1987. Protein import into yeast mitochondria is inhibited by antibodies raised against $45 \mathrm{Kd}$ proteins of the outer membrane. EMBO J. 6:2109-2115.

20.Ølsvik, O., T. Popovic, E. Skjerve, K.S. Cudjoe, E. Hornes, J. Ugelstad and M. Uhlèn. 1994. Magnetic separation techniques in diagnostic microbiology. Clin. Microbiol. Rev. 7:43-54.

21.Pain, D., Y.S. Kanwar and G. Blobel. 1988. Identification of a receptor for protein import into chloroplasts and its localization to envelop contact zones. Nature 331:232-234.

22.Perry, S.E. and K. Keegstra. 1994. Envelope membrane proteins that interact with chloroplast precursor proteins. Plant Cell 6:93-105.

23.Rasmussen, A.-M., E. Smeland, B.K. Erikstein, L. Caignault and S. Funderud. 1992. A new method for detachment of Dynabeads from positively selected B lymphocytes. J. Immunol. Methods 146:195-202.

24.Schnell, D.J., F. Kessler and G. Blobel. 1994. Isolation of components of the chloroplast import machinery. Science 266:1007-1012.

25.Stoppin, V., M. Vantard, A.C. Schmit and A.M. Lambert. 1994. Isolated plant nuclei nu- cleate microtubule assembly: the nuclear surface in higher plants has centrosome-like activity. Plant Cell 6:1099-1106.

26.Tranel, P.J., J. Froehlich, A. Goyal and K. Keegstra. 1995. A component of the chloroplast protein import apparatus is targeted to the outer envelope membrane via a novel pathway. EMBO J. 14:2436-2446.

27.Walbot, V. and D.A. Hoisington. 1982. Isolation of mesophyll and bundle sheath chloroplasts for maize, p. 211-219. In M. Edelman, R.B. Hallick and N.-H. Chua (Eds.), Methods in Chloroplast Molecular Biology. Elsevier Biomedical Press, Amsterdam.

28.Yoneda, Y., N. Imamoto-Sonobe, Y. Matsuoka, R. Iwamoto, Y. Kiho and T. Uchida. 1988. Antibodies to Asp-Asp-Glu-Asp can inhibit transport of nuclear proteins into the nucleus. Science 242:275-278.

Received 11 August 1998; accepted 5 November 1998.

Address correspondence to:

Dr. Barry D. Bruce

Department of Biochemistry, Cellular and

Molecular Biology

Walters Life Science Building

University of Tennessee at Knoxville

Knoxville, TN 37996, USA

Internet: bbruce@utk.edu 